\title{
openheart Founder mutation in myosin-binding protein $C$ with an early onset and a high penetrance in males
}

Irene Méndez (10 , ,2,3,4 Ana Isabel Fernández, ${ }^{2,3,4}$ Maria Ángeles Espinosa, ${ }^{1,2,3,4}$ Sofía Cuenca, ${ }^{1,2,3,4}$ Rebeca Lorca, , ${ }^{1,3,4}$ José Fernando Rodríguez, ,2,3,4 Maria Tamargo, ,2,3,4 Marta García-Montero (D) , 1,2,3,4 Cristina Gómez, ${ }^{1,2,3,4}$ Silvia Vilches, ${ }^{1,2,3,4}$ Nélida Vázquez, ${ }^{1}$ Reyes Álvarez, ${ }^{3,4,5}$ Constancio Medrano, ${ }^{3,4,5}$ Raquel Yotti, ${ }^{6}$ Francisco Fernández-Avilés, ${ }^{1,2,3,4}$ Javier Bermejo (1) 1,2,3,4

- Additional supplemental material is published online only. To view, please visit the journal online (http://dx.doi.org/10. 1136/openhrt-2021-001789).

To cite: Méndez I, Fernández Al, Espinosa MÁ, et al. Founder mutation in myosin-binding protein $\mathrm{C}$ with an early onset and a high penetrance in males. Open Heart 2021;8:e001789. doi:10.1136/ openhrt-2021-001789

Received 15 July 2021 Accepted 30 August 2021

Check for updates

\section{(c) Author(s) (or their} employer(s)) 2021. Re-use permitted under CC BY-NC. No commercial re-use. See rights and permissions. Published by BMJ.

${ }^{1}$ Cardiology, Hospital General Universitario Gregorio Marañón, Madrid, Spain

${ }^{2}$ CIBERCV, Madrid, Spain ${ }^{3}$ Instituto de Investigación Sanitaria Gregorio Marañón, Madrid, Spain

${ }^{4}$ Facultad de Medicina, Universidad Complutense, Madrid, Spain

${ }^{5}$ Pediatric Cardiology, Hospital Materno Infantil Gregorio Marañón, Madrid, Spain ${ }^{6}$ Instituto de Salud Carlos III, Madrid, Spain

Correspondence to Dr Irene Méndez; imendezfdez@ gmail.com

\section{ABSTRACT}

Objective One of the challenges in hypertrophic cardiomyopathy $(\mathrm{HCM})$ is to determine the pathogenicity of genetic variants and to establish genotype/phenotype correlations. This study aimed to: (1) demonstrate that MYBPC3 c.2149-1G $>\mathrm{A}$ is a founder pathogenic variant, (2) describe the phenotype and clinical characteristics of mutation carriers and (3) compare these patients with those with the most frequent pathogenic HCM variants: MYBPC3 p.Arg502Trp/Gln.

Methods We reviewed genetic tests performed in HCM probands at our institution. We carried out transcript analyses to demonstrate the splicing effect, and haplotype analyses to support the founder effect of MYBPC3 c.2149-1G $>$ A. Carriers with this mutation were compared with those from MYBPC3 p.Arg502Trp/GIn in terms of presentation features, imaging and outcomes. Results MYBPC3 c.2149-1G>A was identified in 8 of 570 probands and 25 relatives. Penetrance was age and sex dependent, $50.0 \%$ of the carriers over age 36 years and $75.0 \%$ of the carriers over 40 years showing HCM. Penetrance was significantly higher in males: in carriers older than 30 years old, $100.0 \%$ of males vs $50.0 \%$ of females had a HCM phenotype $(p=0.01)$. Males were also younger at diagnosis ( $32 \pm 13$ vs $53 \pm 10$ years old, $\mathrm{p}<0.001)$. MYBPC3 c.2149-1G>A resulted in an abnormal transcript that led to haploinsufficiency and was segregated in two haplotypes. However, both came from one founder haplotype. Affected carriers showed a better functional class and higher left ventricular ejection fraction (LVEF) than patients with MYBPC3 p.Arg502Trp/GIn ( $<<0.05$ for both). Nevertheless, the rate of major adverse outcomes was similar between the two groups.

Conclusions MYBPC3 c.2149-1G $>$ A splicing variant is a founder mutation. Affected males show an early onset of HCM and with higher penetrance than women. Carriers show better functional class and higher LVEF than MYBPC3 p.Arg502Trp/GIn carriers, but a similar rate of major adverse outcomes.

\section{INTRODUCTION}

Hypertrophic cardiomyopathy (HCM) is the most common inherited cardiac disease with

Key questions
What is already known about this subject?
Hypertrophic cardiomyopathy (HCM) is the most
common inherited cardiac disease, and it is genet-
ically and clinically heterogeneous. Thousands of
pathogenic variants causing HCM have been de-
scribed but few genotype-phenotype correlations
have been established because most of the patho-
genic variants are unique or seen in a limited num-
ber of families.
What does this study add?
This study shows that MYBPC3 c.2149-1G>A is a
founder mutation that alters splicing, changes the
reading frame and causes a truncated protein. The
cosegregation of MYBPC3 c.2149-1G>A in eight
non-related HCM families confirms the pathogenici-
ty of the variant. Most MYBPC3 c.2149-1G>A carri-
ers present a moderate left ventricular hypertrophy,
but a highly variable expressivity is observed. The
incidence of major adverse outcomes is similar to
other HCM variants. Male carriers show an earlier
onset and a higher penetrance than female carriers.
How might this impact on clinical practice?
genicity permits restricting the follow-up of relatives
to mutation carriers. These will need lifetime sur-
veillance to early identify the development of dis-
ease and treat and prevent its complications.
the

an estimated prevalence of 1 in 500 individuals. ${ }^{1}$ HCM is a heterogeneous disease with a wide clinical spectrum, characterised by increased left ventricular wall thickness (LVWT) in the absence of abnormal loading conditions. ${ }^{12}$ Although many patients have asymptomatic or mildly symptomatic forms and normal life expectancy, other patients develop malignant phenotypes associated with sudden cardiac death (SCD) and end stage heart failure. Atrial fibrillation (AF) 
and left ventricular outflow tract obstruction (LVOTO) are additional common complications. ${ }^{3} \mathrm{HCM}$ is inherited with an autosomal dominant pattern, with incomplete and age dependent penetrance. A pathogenic variant in a sarcomere gene is found in about $35 \%-50 \%$ of HCM cases and MYBPC 3 is the most frequent affected gene. ${ }^{4-6}$

More than 2000 pathogenic variants in sarcomeric genes have been described until date. Most of them have a very low frequency in HCM cohorts or are confined to individual families. ${ }^{37}$ An exception is the $M Y B P C 3$ p.Arg502Trp variant (c.1504G $>\mathrm{T})$. Representing 1.5\%-3\% of HCM patients, this mutation is the most frequent in different HCM cohorts. ${ }^{48}$ Two other missense pathogenic variants have been described at this position, p.Arg502Gln (c.1505G >A) and p.Arg502Leu (c.1505G >T).$^{9}$

Few specific genotype-phenotype correlations have been demonstrated in HCM, probably because most of the pathogenic variants are unique or seen in limited numbers. However, when founder pathogenic mutations are identified, their study represents a unique opportunity to describe clinical phenotypes. ${ }^{10-19}$ The $M Y B P C 3$ c.2149-1G $>\mathrm{A}$ variant has been previously described in a single HCM proband, without clinical and segregation data. ${ }^{20}$ In the ClinVar public database, this variant is reported in other three non-related HCM patients, but there is still no clinical or segregation information available. The sequence change affects an acceptor splice site, so it is expected to result in an absent or disrupted protein. However, no functional analyses have been conducted. The main objectives of this study were (1) to address whether the identified MYBPC3 c.2149-1G>A mutation is a founder pathogenic variant, (2) clarify its functional consequences and (3) to describe clinical characteristics and phenotype of affected carriers. In addition, we compared the phenotype and clinical profile of patients with this mutation with a control group of MYBPC3 p.Arg502Trp/p.Arg502Gln carriers, the most frequent HCM pathogenic variants in our institution.

\section{METHODS}

\section{Study population and genetic analysis}

We reviewed all genetic analyses in HCM probands performed in our institution between January 2012 and August 2019, screening for MYBPC3 variants. Genetic analyses in HCM probands were performed by Sanger sequencing of the five main sarcomeric genes (MYBPC3, MYH7, TNNT2, TNNI3 y TPM1) between January and November 2012, and by next-generation sequencing of a panel of 25 HCM-related genes from that date onwards. All patients signed an informed consent document authorising the use of their genetic data for research purposes.

\section{Patient and public involvement}

Patients were not involved in the design, development of the study or the interpretation and writing of the results.

\section{Clinical evaluation}

Following current recommendations, ${ }^{1}{ }^{2}$ the diagnosis of HCM was established by a left ventricular hypertrophy $\geq 15 \mathrm{~mm}$ in probands and $\geq 13 \mathrm{~mm}$ in relatives. A pedigree was elicited for each proband, and relatives were offered clinical and genetic screening if a pathogenic or likely pathogenic mutation was identified in the proband. HCM patients were followed up and treated according to current recommendations. We defined LVOTO as the presence of a left ventricle outflow tract pressure gradient $\geq 30 \mathrm{~mm} \mathrm{Hg}$ at rest. A pressure gradient $\geq 50 \mathrm{~mm}$ $\mathrm{Hg}$ was considered haemodynamically relevant. ${ }^{12}$ All HCM patients, whether they were gene positive or negative, underwent arrhythmic risk stratification based on the presence of recognised arrhythmic risk factors for HCM. Since 2014, the 5-year risk of SCD was estimated according to the European Society of Cardiology (ESC) score. ${ }^{121}$ Any episode of ischaemic stroke, SCD, aborted sudden cardiac arrest (SCA), appropriate implantable cardioverter defibrillator (ICD) shock, heart failure admission, cardiac transplantation or cardiac death was recorded as a major adverse outcome.

\section{Cardiovascular magnetic resonance}

All probands and relatives that fulfilled the HCM criteria at first evaluation were offered scanning by cardiovascular MR (CMR). Patients underwent CMR with a 1.5-T Philips Achieva scanner. The CMR study consisted of cine steady-state free-precession imaging of left ventricular function and late enhancement imaging of myocardial scar tissue (3D inversion-recovery turbo gradient echo sequence). Images were obtained in short-axis views and four-chamber, two-chamber and three-chamber views. Late gadolinium enhancement (LGE) was performed $10 \mathrm{~min}$ after a total injection of $0.2 \mathrm{mmol} / \mathrm{kg}$ gadoteridol. CMR data were analysed by investigators blinded to patient's genotype, using dedicated software (QMass MR V.8.1, MEDIS Suite V.3.2). Left ventricular end-diastolic and end-systolic volumes, ejection fraction and mass were measured from short axis views. ${ }^{22}$ The presence of LGE was determined by visual inspection. Scar size (extent) was calculated from LGE sequences as a percentage of myocardium by with semi-automated planimetry (manually corrected) using full-width half-max thresholding.

\section{Splicing analysis}

In silico analysis of $M Y B P C 3$ c.2149-1G $>\mathrm{A}$ was performed using Human Splicing Finder (http://www.umd.be/ HSF/HSF.shtml) and MaxEntScan (http://hollywood. mit.edu/burgelab/maxent/Xmaxentscan_scoreseq. html) bioinformatic prediction tools. Additionally, RNA isolated from blood samples of one MYBPC3 c.2149$1 \mathrm{G}>\mathrm{A}$ carrier and one non $M Y B P C 3$ c.2149-1G $>\mathrm{A}$ carrier was analysed by PCR using a primer pair from exon 22 to exon 24 (Fw: 5'-AGCCCCAGATGCCCCAGAGGA-3'and Rv: 5'-GTAGGCAGGCGGCTCCCACTGTA-3'), to validate transcript sizes. 


\section{Ancestor analysis}

To identify the haplotypes sharing $M Y B P C 3$ c.2149-1G $>\mathrm{A}$ variant, six markers: rs7120013, rs4882135, rs671299, rs7116652, rs10792299 and rs11605489 (online supplemental table S1) and MYBPC3 c.2149-1G>A variant were genotyped by Sanger sequencing in available samples, 16 MYBPC3 c.2149-1G $>$ A carriers and 6 non-carriers. These markers covered from 46 to $62 \mathrm{Mb}$ of chromosome 11 , and segregate at intermediate frequency at population level (selected from the 1000 Genomes Browser database, www.ncbi.nlm.nih.gov/variation/tools/1000genomes/). Genotype information for those markers was extracted for an Iberian population $(n=107)$ from the 1000 Genomes Browser database. Haplotypes were reconstructed employing family information and the Iberian population genotype data to infer most probable haplotypes using phase V.2.1 software. $^{23}$ Reconstructed haplotypes were subjected to phylogenetic analysis using minimum evolution, bootstrap (1000 replicates) and tree neighborjoining in MEGA V.7 software. ${ }^{24}$

\section{Statistical analysis}

Data are expressed as mean $\pm \mathrm{SD}$ and frequencies or percentages where appropriate. We used ordinary leastsquare linear regression to compare continuous variables, and $\chi^{2}$ to compare qualitative variables. The penetrance, diagnosis age and cumulative probability of an event on follow-up was estimated using the Kaplan-Meier method. We used log-rank tests and Cox proportional-hazards models to compare survival between groups (MYBPC3 variant and gender). A p $<0.05$ was considered statistically significant.

\section{RESULTS}

\section{Study population}

A total of $570 \mathrm{HCM}$ probands underwent genetic testing at our institution between 2012 and 2019. A MYBPC3 pathogenic variant was identified in 79 (14.0\%). MYBPC3 p.Arg502Gln (c.1505G>A, NM_00256.3) and MYBPC3 p.Arg502Trp (c.1504C>T, NM_00256.3) were the most frequent variants detected in $M Y B P C 3$, identified in heterozygosis in 16 unrelated HCM probands $(2.8 \%$ of the HCM probands) and 17 relatives (8 were affected, $47.0 \%$ ). The MYBPC3 c.2149-1G $>$ A (NM_00256.3) variant was identified in eight unrelated HCM probands (all male), representing $1.4 \%$ of all probands. We studied 45 family members and identified 25 MYBPC3 c.2149$1 \mathrm{G}>\mathrm{A}$ carriers, $12(48.0 \%)$ of them with HCM phenotype and $13(52.0 \%)$ unaffected carriers (figure 1).

\section{Penetrance and age at diagnosis}

Overall penetrance in MYBPC3 c.2149-1G>A carriers was $62.0 \%$ and it was age dependent: $50.0 \%$ of the carriers over age 36 years and $75.0 \%$ of the carriers over 40 years had HCM phenotype. Moreover, in male patients older than 30 years, penetrance was 13/13 (100.0\%), significantly higher than in females above this age
$(5 / 10,50.0 \%, p=0.01)$. The mean age at diagnosis was $37 \pm 15$ years, significantly lower in males than in females $(32 \pm 13$ vs $53 \pm 10$, respectively, $\mathrm{p}<0.001)$. There were no significant differences on penetrance and age at diagnosis compared with MYBPC3 p.Arg502Trp/Gln carriers (log-rank $\mathrm{p}=0.16$ and $\mathrm{p}=0.60$ for penetrance and age at diagnosis, respectively). For MYBPC3 p.Arg502Trp/Gln, age at diagnosis was also lower in males $(28 \pm 20$ vs $50 \pm 19$ years old, $\mathrm{p}=0.007)$. However, as opposed to MYBPC3 c.2149-1G $>$ A carriers, no gender differences $(p=0.08)$ on penetrance were observed for carriers of this control variant (table 1 and figure 2 ).

\section{Phenotype, clinical characteristics and outcomes}

Phenotype and clinical data from 20 identified $M Y B P C 3$ c.2149-1G $>$ A carriers were used for comparison. The reason for diagnosis were symptoms in 6 patients $(30.0 \%)$, and familiar screening or casual finding in $14(70.0 \%)$. At first evaluation, 19 affected carriers (95.0\%) showed asymmetric left ventricular hypertrophy affecting the anterior septum, only one patient presented a concentric LVH pattern. Mean LVWT was $21 \pm 6 \mathrm{~mm}$ and $19 \pm 5 \mathrm{~mm}$ measured by echocardiography and CMR, respectively. The left atrium was enlarged in 12 of $20(60.0 \%)$ affected carriers. LVOTO at rest was detected in three individuals at diagnosis, being haemodynamically relevant in two cases, although to date none of them has required invasive management (table 1). CMR was performed in 18 of the 20 affected carriers, showing LGE in 15 of them $(83 \%)$. The three patients without LGE were young patients with mild phenotypes (maximum LVWT $\leq 16 \mathrm{~mm}$ ). Mean LV mass was $65 \pm 29 \mathrm{~g}$ and mean percentage of fibrosis was $16 \% \pm 14$, with $35.0 \%$ of patients showing a percentage of fibrosis $>15 \%$ (table 2 ).

A great variability on phenotype was detected in patients carrying $M Y B P C 3$ c.2149-1G $>$ A that varied from normal phenotype to severe disease with extensive fibrosis or even restrictive phenotype (figure $3 \mathrm{~A}-\mathrm{H}$ ).

Follow-up data for MYBPC3 c.2149-1G>A affected patients was available for a median of 9 (range 1-34) years since diagnosis. After this period, 19 patients $(95.0 \%)$ remained mildly symptomatic (New York Heart Association I-II). Four patients (20.0\%, 4 males) developed AF. At least an episode of non-sustained ventricular tachycardia was detected in 7 patients (35.0\%). Nine patients $(45.0 \%)$ had a major classical SCD risk factor (six had a family history of SCD, two had LVWT $\geq 30 \mathrm{~mm}$, one had a syncope). The ESC risk score was higher than $4 \%$ in eight carriers $(40.0 \%)$. These eight patients were offered an ICD which was finally implanted in seven $(35.0 \%)$ because of patient's preferences. One of the ICD implants took place in secondary prevention after an aborted SCA, as the patient had also previously rejected ICD implantation (table 1).

There were six major adverse outcomes registered: one patient suffered an aborted SCA and posteriorly appropriate ICD shocks, and another patient had appropriate ICD shocks. Two patients were admitted for heart failure 
Family $\mathrm{A}$

I

II<smiles>CO</smiles>

II

IV

Family C

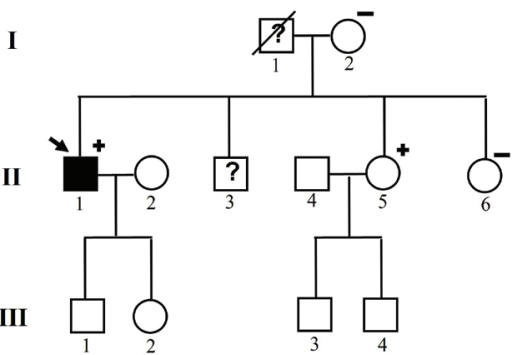

$\triangle_{1} \oslash_{2}$

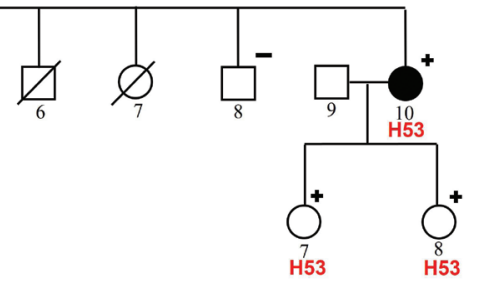

Family D

I

II

III

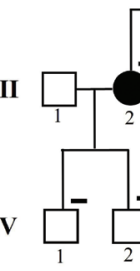

Family B

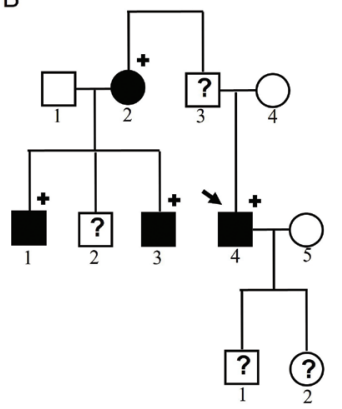

Family $\mathrm{F}$
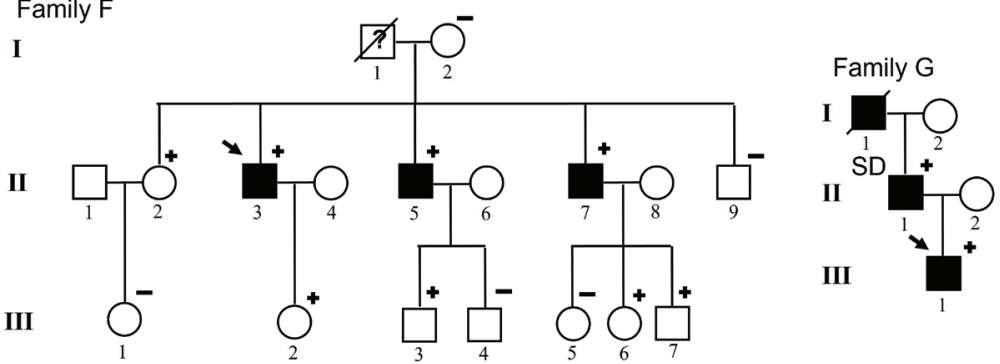

Family $\mathrm{H}$
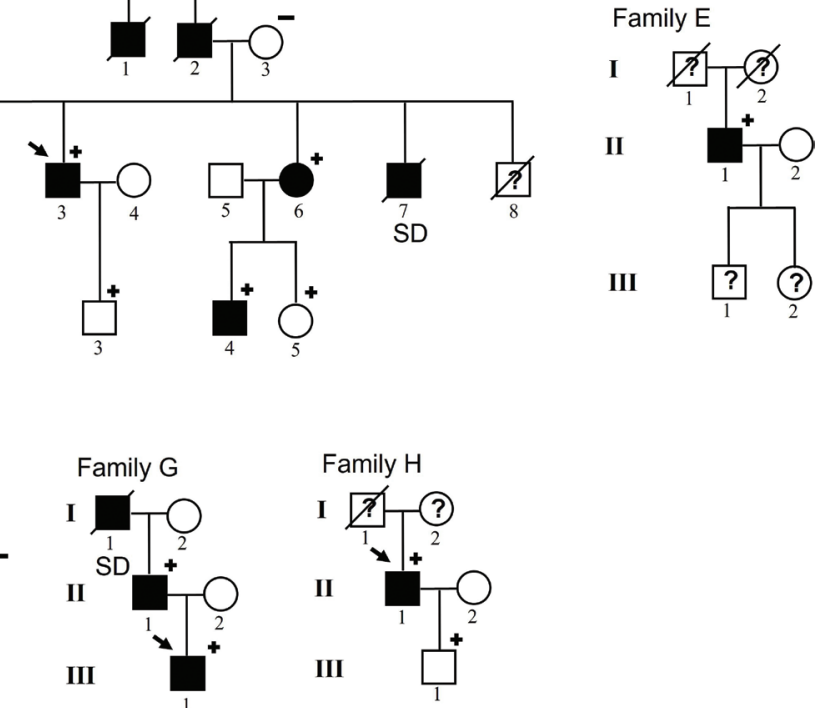

Figure 1 MYBPC3 c.2149-1G>A pedigrees. Symbols denote sex, genetic and disease status: +, carriers; -, non-carriers; ?, unknown phenotype; box, male; circle, female; darkened, affected; slashed, deceased; clear symbol, unaffected; without sign, not studied. SD, sudden death.

and another patient with a restrictive phenotype developed advanced heart failure and underwent evaluation for cardiac transplantation (table 3). The rate of major adverse outcomes was $0.82 \%$ per year.

Compared with MYBPC3 p.Arg502Trp/Gln, MYBPC3 c.2149-1G>A patients had higher left ventricular ejection fraction (LVEF) $(\mathrm{p}=0.03)$ and better functional class $(p=0.04$; table 1$)$. No significant differences for CMR measures were identified between both $M Y B P C 3$ variants (table 2). Survival analysis showed similar outcomes in both patient groups (HR 0.3, (95\% CI 0.3 to 4.9$) \mathrm{p}=0.706$; table 3 and figure 4 ).

\section{Splicing analysis}

HSF and MaxEntScan tools predicted that MYBPC3 c.2149-1G $>$ A alters the acceptor splice site producing an abnormal splicing. In this case, intron between exon 22 and 23 would skip splicing and would be included in the mature RNA, changing the reading frame and causing a truncated protein. Transcript analysis confirmed that MYBPC3 c.2149-1G>A generated an abnormal transcript (online supplemental figure S1).

\section{Ancestor analysis}

The haplotype reconstruction showed 53 different haplotypes in the Iberian population. The most probable segregation for $M Y B P C 3$ c.2149-1G>A carriers was estimated in two haplotypes, H52: GATACGA and H53: AATACGA. The phylogeny reconstruction estimated that both H52 and H53 may come from common ancestor H16 (figure 5A). In fact, H52 may come from one recombination event in $\mathrm{H} 53$ haplotype that has recently occurred in one of the families (figure 5B).

\section{DISCUSSION}

To our knowledge, this is the first study to describe the clinical phenotype of the MYBPC3 c.2149-1G>A variant, herein demonstrating cosegregation in eight non-related HCM families (figure 1). This information confirms the pathogenicity of the variant. Hence, this variant can be classified as a definite pathogenic variant with strong evidence according to the American College of Medical Genetics criteria. ${ }^{25}$ This information is essential for patient management because it enables 
Table 1 MYBPC3 c.2149-1G>A and MYBPC3 p.Arg502Tr/Gln comparison

\section{MYBPC3}

c.2149-1G>A

MYBP3

\begin{tabular}{llllllll}
\hline Male & Female & Total & & Male & Female & Total & P value \\
15 & 5 & 20 & 17 & 7 & 24 &
\end{tabular}

\begin{tabular}{|c|c|c|c|c|c|c|c|}
\hline N & 15 & 5 & 20 & 17 & 7 & 24 & \\
\hline Age at diagnosis (mean $\pm S D$, years) & $32 \pm 13$ & $53 \pm 10$ & $37 \pm 15$ & $28 \pm 20$ & $50 \pm 19$ & $35 \pm 21$ & $0.650^{*}$ \\
\hline Max. LVWT TTE (mean $\pm S D$, mm) & $21 \pm 6$ & $22 \pm 6$ & $21 \pm 6$ & $21 \pm 7$ & $21 \pm 6$ & $21 \pm 7$ & 0.645 \\
\hline LVEF (mean $\pm S D, \%)$ & $63 \pm 9$ & $62 \pm 10$ & $63 \pm 9$ & $54 \pm 15$ & $51 \pm 16$ & $54 \pm 15$ & 0.032 \\
\hline Left atrial diameter (mm) & $44 \pm 9$ & $42 \pm 8$ & $44 \pm 9$ & $41 \pm 8$ & $44 \pm 8$ & $42 \pm 8$ & 0.210 \\
\hline Severe LVOTO $\geq 50$ mm Hg (\%) & $2(13.3)$ & 0 & $2(10.0)$ & $2(11.7)$ & 0 & $2(8.4)$ & 0.800 \\
\hline LGE $(\%)$ & $10(66.7)$ & $5(100.0)$ & $15(75.0)$ & $12(70.6)$ & $6(85.7)$ & $18(75.0)$ & 0.550 \\
\hline Diagnostic reason & & & & & & & 0.242 \\
\hline Symptoms (\%) & $6(40.0)$ & 0 & $6(30.0)$ & $5(29.4)$ & $2(28.6)$ & $7(29.2)$ & \\
\hline Aborted cardiac arrest (\%) & 0 & 0 & 0 & $1(5.9)$ & 0 & $1(4.2)$ & \\
\hline Asymptomatic (\%) & $9(60.0)$ & $4(80.0)$ & $13(65.0)$ & $12(70.6)$ & $3(42.1)$ & $15(62.5)$ & \\
\hline Dyspnoea (\%) & $2(13.3)$ & $1(20.0)$ & $3(15.0)$ & $4(23.5)$ & $2(28.6)$ & $6(25.0)$ & \\
\hline Syncope (\%) & $1(6.7)$ & 0 & $1(5.0)$ & 0 & 0 & 0 & \\
\hline Chest pain (\%) & 0 & 0 & 0 & $1(5.9)$ & 0 & $1(4.1)$ & \\
\hline Palpitations (\%) & $3(20.0)$ & 0 & $3(15.0)$ & 0 & 0 & 0 & \\
\hline NYHA functional class & & & & & & & 0.049 \\
\hline I (\%) & $11(73.3)$ & $4(80.0)$ & $15(75.0)$ & $7(41.2)$ & $1(14.3)$ & $8(33.3)$ & \\
\hline || (\%) & $3(20.0)$ & $1(20.0)$ & $4(20.0)$ & $7(41.2)$ & $4(57.1)$ & $11(45.9)$ & \\
\hline$>6(\%)$ & $3(20.0)$ & 0 & $3(15.0)$ & $2(11.7)$ & 0 & $2(8.4)$ & 0.300 \\
\hline ICD indication & & & & & & & 0.874 \\
\hline Primary prevention (\%) & $5(33.3)$ & $1(20.0)$ & $6(30.0)$ & $6(35.3)$ & $3(42.1)$ & $9(37.5)$ & \\
\hline Secondary prevention (\%) & $1(6.7)$ & 0 & $1(5.0)$ & $2(11.7)$ & 0 & $2(8.4)$ & \\
\hline
\end{tabular}

*Log-rank estimate.

ABPR, abnormal blood pressure response to exercise; CMR, cardiac MR; ICD, implantable cardioverter defibrillator; LGE, late gadolinium enhancement; LVEF, left ventricle ejection fraction; LVOTO, left ventricular outflow tract obstruction; LVWT, left ventricular wall thickness; NSVT, non-sustained ventricular tachycardia; ; NYHA, New York Heart Association; SCD, sudden cardiac death; TTE, transthoracic echocardiography.

to perform genetic screening in family members and narrows the clinical follow-up to be performed only in carriers.

\section{Mechanism of pathogenicity and founder effect}

MYBPC3 c.2149-1G>A alters the canonical donor splice sequence, so it is supposed to generate alternative splicing. However, to our knowledge, no functional studies on this mutation are available. This is the first time that RNA analysis is performed, demonstrating that this variant alters normal splicing. This $\mathrm{G}>\mathrm{A}$ change triggers intron 22-23 retention, causing a frameshift that leads to a truncated protein. Unlike other sarcomeric genes, where the majority of pathogenic variants are missense with a dominant negative effect, up to $60 \%$ of the pathogenic variants in $M Y B P C 3$ are frameshifts leading to haploinsufficiency. ${ }^{26}$ In fact, all the founder pathogenic 

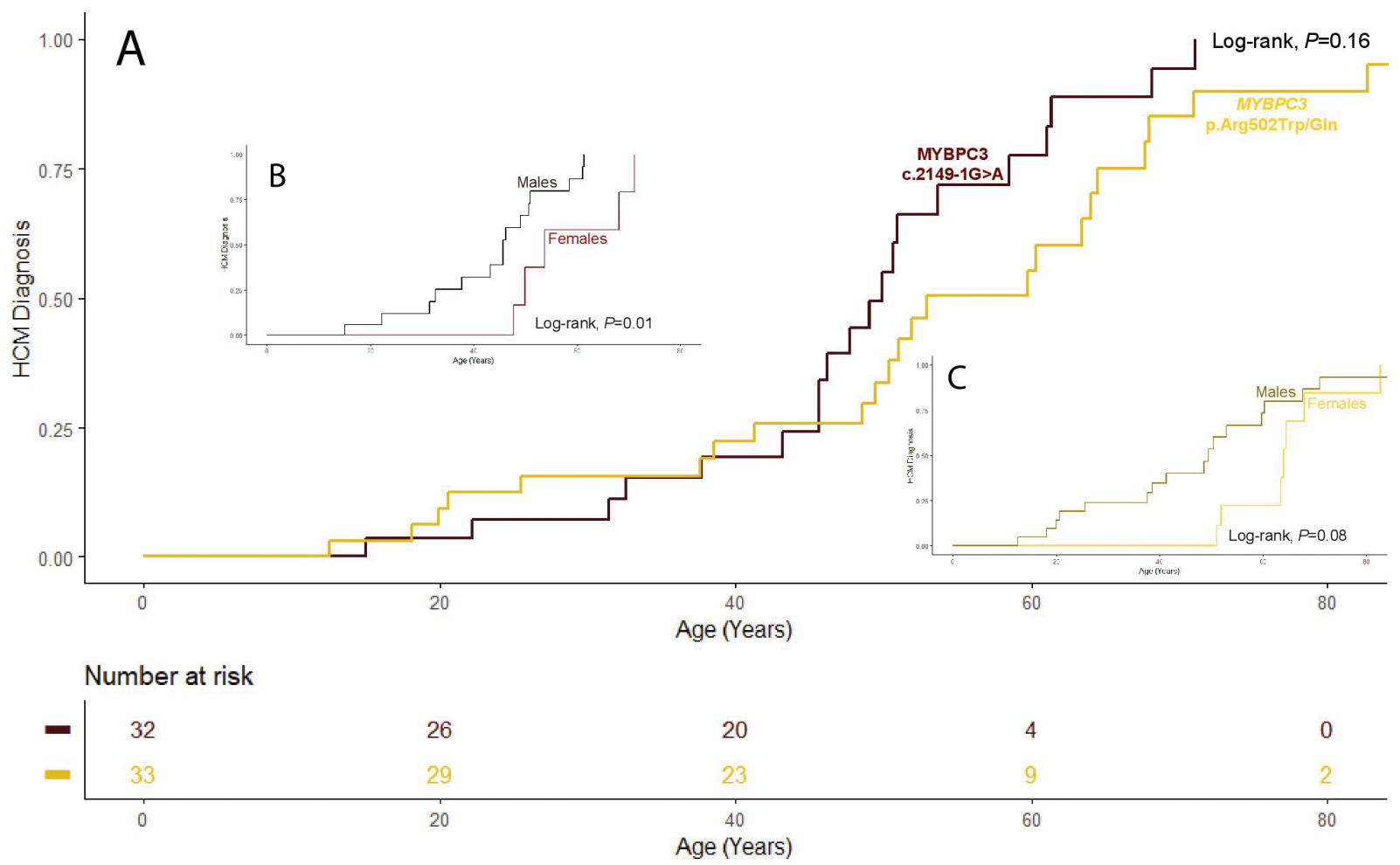

Figure 2 Penetrance. Comparison between MYBP3 c.2149-1G>A and MYBPC3 p.Arg502Trp/Gln variants and between sex for each variant. (A) Full study population. (B) MYBP3 c.2149-1G>A sex analysis. (C) MYBPC3 p.Arg502Trp/Gln sex analysis. HCM, hypertrophic cardiomyopathy.

variants previously described in $M Y B P C 3$ are truncated variants. ${ }^{10-19}$ On the other hand, the mechanisms of pathogenicity of MYBPC3 p.Arg502Trp and p.Arg502Gln are unclear. Previous studies have shown that MYBPC3 p.Arg502Trp alters the predicted electrostatic properties of the C3 domain of the protein and may directly disrupt the interaction of MYBPC3 with other sarcomeric proteins. ${ }^{27}$

The haplotype analyses performed in our study supports a founder origin of MYBPC3 c.2149-1G>A, as all the analysed carriers, related and unrelated, shared the same haplotype along $16 \mathrm{Mb}$. Actually, two haplotypes carrying MYBPC3 c.2149-1G $>$ A were identified in one single family, which likely corresponds to a recent recombination event as all other family members share the same MYBPC3 c.2149-1G>A haplotype. All carriers are from the same geographical area, the South region of Madrid (Spain). The opposite to founder mutation occurs with $M Y B P C 3$ p.Arg502Trp variant, where several different haplotypes appear segregating with the variant. ${ }^{9}$ Therefore, the high frequency of this variant seems to be explained by recurrent mutation of this residue rather than a founder effect. Further evidence that supports that MYBPC3 codon 502 may be a mutational hotspot is the existence of two other HCM mutations that alter

\begin{tabular}{|c|c|c|c|c|c|c|c|}
\hline & \multicolumn{3}{|c|}{$\begin{array}{l}\text { МУВРC3 } \\
\text { c.2149-1G>A }\end{array}$} & \multicolumn{3}{|c|}{$\begin{array}{l}\text { MYBP3 } \\
\text { p.Arg502Trp/GIn }\end{array}$} & \multirow{2}{*}{$\begin{array}{l}P \\
\text { value }\end{array}$} \\
\hline & Male & Female & Total & Male & Female & Total & \\
\hline N & 10 & 4 & 14 & 12 & 6 & 18 & \\
\hline LVEDV (mean $\pm S D, m L)$ & $188 \pm 47$ & $144 \pm 18$ & $175 \pm 45$ & $195 \pm 57$ & $178 \pm 39$ & $190 \pm 51$ & 0.407 \\
\hline LVESV (mean $\pm S D, m L)$ & $83 \pm 26$ & $55 \pm 18$ & $75 \pm 27$ & $98 \pm 33$ & $86 \pm 53$ & $94 \pm 39$ & 0.128 \\
\hline $\mathrm{EF}($ mean $\pm \mathrm{SD}, \%)$ & $56 \pm 12$ & $63 \pm 8$ & $58 \pm 11$ & $49 \pm 14$ & $55 \pm 17$ & $51 \pm 15$ & 0.163 \\
\hline LV mass (mean $\pm S D, g)$ & $151 \pm 47$ & $91 \pm 15$ & $134 \pm 48$ & $161 \pm 72$ & $103 \pm 16$ & $142 \pm 65$ & 0.669 \\
\hline LV mass index (mean $\pm S D, g$ per $\mathrm{m}^{2}$ ) & $78 \pm 36$ & $50 \pm 6$ & $65 \pm 29$ & $84 \pm 36$ & $53 \pm 5$ & $77 \pm 34$ & 0.399 \\
\hline Fibrotic mass (mean $\pm S D, g)$ & $38 \pm 49$ & $12 \pm 7$ & $31 \pm 43$ & $39 \pm 42$ & $37 \pm 26$ & $38 \pm 36$ & 0.620 \\
\hline Fibrotic mass index $\left(\right.$ mean $\pm S D, g$ per $\left.\mathrm{m}^{2}\right)$ & $27 \pm 39$ & $7 \pm 5$ & $18 \pm 30$ & $20 \pm 21$ & $22 \pm 21$ & $21 \pm 20$ & 0.798 \\
\hline$\%$ fibrosis $($ mean $\pm \mathrm{SD}, \%)$ & $17 \pm 17$ & $12 \pm 6$ & $16 \pm 14$ & $20 \pm 14$ & $28 \pm 17$ & $23 \pm 15$ & 0.195 \\
\hline
\end{tabular}

LVEDV, left ventricular end-diastolic volume; LVESV, left ventricular end-systolic volume; EF, ejection fraction. 


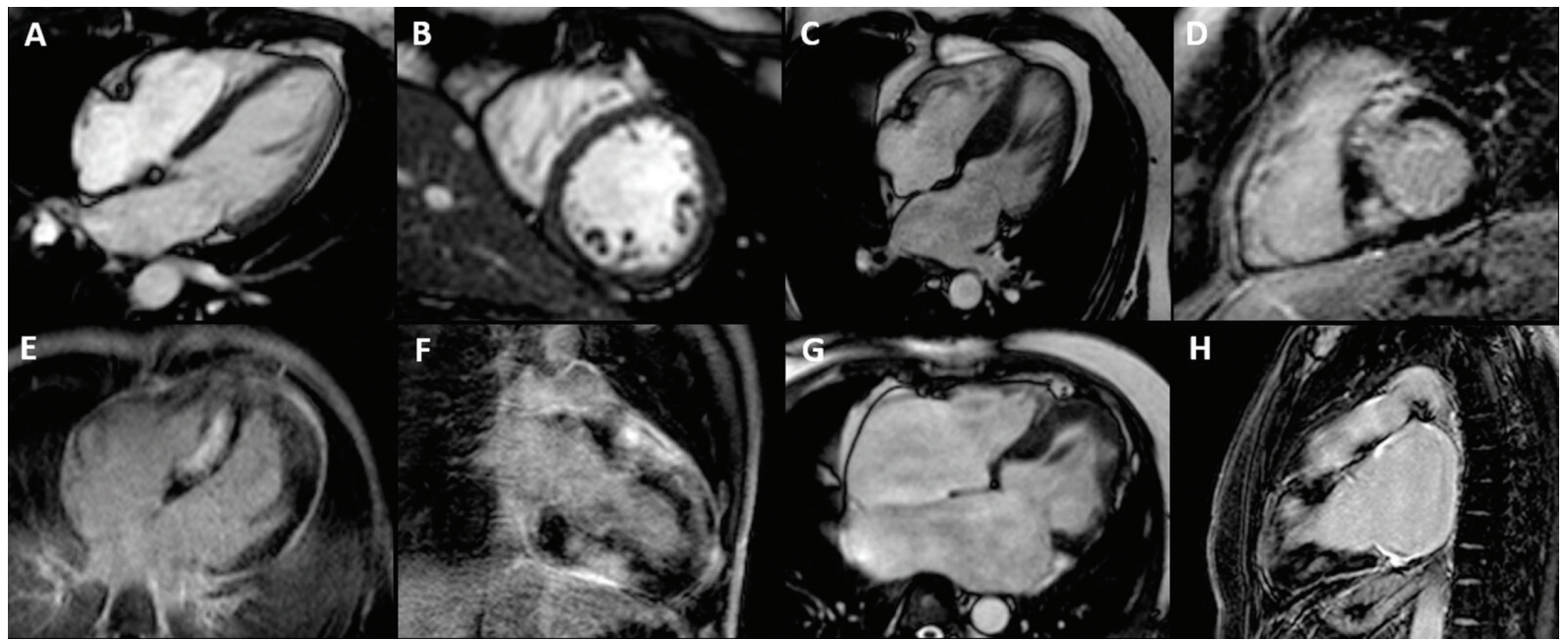

Figure 3 Cardiac MR (CMR) images showing phenotype variability in MYBPC3 c.2149-1G>A carriers. (A-D) CMR images of two brothers: A, B is a 48-year-old female carrier with normal phenotype and C, D is her 49-year-old affected brother with septal hypertrophy of $22 \mathrm{~mm}$ and extensive LGE. (E, F) CMR images showing a severe left ventricle hypertrophy with extensive LGE in a 21-year-old carrier with severe systolic disfunction. (G, H) CMR images showing a restrictive phenotype with severe atrial enlargement and fibrosis. LGE, late gadolinium enhancement.

arginine 502 to either glutamine or to leucine and the fact that variants at this position are frequent in HCM cohorts from different countries. ${ }^{4-9}$

\section{Penetrance, phenotype and clinical characteristics}

Although the penetrance of MYBPC3 c.2149-1G $>\mathrm{A}$ was incomplete and age-dependent as in most $M Y B P C 3$ variants, penetrance was particularly high in young males, reaching $100.0 \%$ in carriers older than 30 years, and significantly higher in males than females. Also, males were younger at the time of diagnosis. Similar gender differences have been described for other $M Y B P C 3$ variants. ${ }^{17-1928}$ This delay in developing the disease in women may be secondary to genetic and endocrine factors that may influence phenotypic expression. Other suggested explanations are the lack of attention to early clinical signs and fewer indications for medical screening programmes in women. ${ }^{28}$ In our study, all affected female carriers of $M Y B P C 3$ c.2149-1G $>$ A were diagnosed after the age of 40 during family screening and most of them were asymptomatic at diagnosis. Therefore, the older age at diagnosis in females in our cohort is better explained by a later-age onset disease than by a delayed diagnosis.

Our imaging data show that MYBPC3 c.2149-1G $>\mathrm{A}$ patients present an HCM phenotype with left ventricular thickening affecting anterior septum, preserved LVEF, frequent left atrial enlargement and extensive LGE with $35.0 \%$ of patients showing relevant fibrosis. However, expressivity was highly variable, ranging from frequent non-affected carriers all the way to a few patients with a severe phenotype and adverse outcomes. This observation may support the relevance of modifier genes and epigenetics and internal/external environmental factors that still are not well understood. ${ }^{29}$

MYBPC3 c.2149-1G $>\mathrm{A}$ affected carriers showed higher LVEF and a better functional class compared with p.Arg502Trp/Gln affected carriers. However, the rate of

Table 3 Major clinical outcomes in MYBP3 c.2149-1G>A and MYBPC3 p.Arg502Trp/Gln affected carriers

\begin{tabular}{|c|c|c|c|c|c|c|c|}
\hline & $\begin{array}{l}\text { MYBPC } \\
\text { c.2149- }\end{array}$ & & & $\begin{array}{l}\text { MYBP3 } \\
\text { p.Arg5 }\end{array}$ & $/$ GIn & & Loq-rank \\
\hline & Male & Female & Total & Male & Female & Total & $p$ value \\
\hline $\mathrm{N}$ & 15 & 5 & 20 & 17 & 7 & 24 & \\
\hline Appropriate ICD shock & $2(13.3)$ & 0 & $2(10.0)$ & $1(5.8)$ & 0 & $1(4.2)$ & 0.700 \\
\hline Heart failure admission & $1(6.7)$ & $1(20.0)$ & $2(10.0)$ & $1(5.8)$ & $2(28.6)$ & $3(12.5)$ & 0.670 \\
\hline Advanced heart failure & $1(6.7)$ & 0 & $1(5.0)$ & 0 & $1(14.3)$ & $1(4.2)$ & 0.620 \\
\hline Ischaemic stroke & 0 & 0 & 0 & $2(11.8)$ & $1(14.3)$ & $3(12.5)$ & 0.190 \\
\hline Aborted SCA & $1(6.7)$ & 0 & $1(5.0)$ & $2(11.8)$ & 0 & $2(8.3)$ & 0.630 \\
\hline Cardiac death & 0 & 0 & 0 & 0 & $1(14.3)$ & $1(4.2)$ & 0.620 \\
\hline Major events & 5 & 1 & 6 & 7 & 5 & 12 & 0.730 \\
\hline
\end{tabular}

ICD, implantable cardioverter defibrillator; SCA, sudden cardiac arrest. 


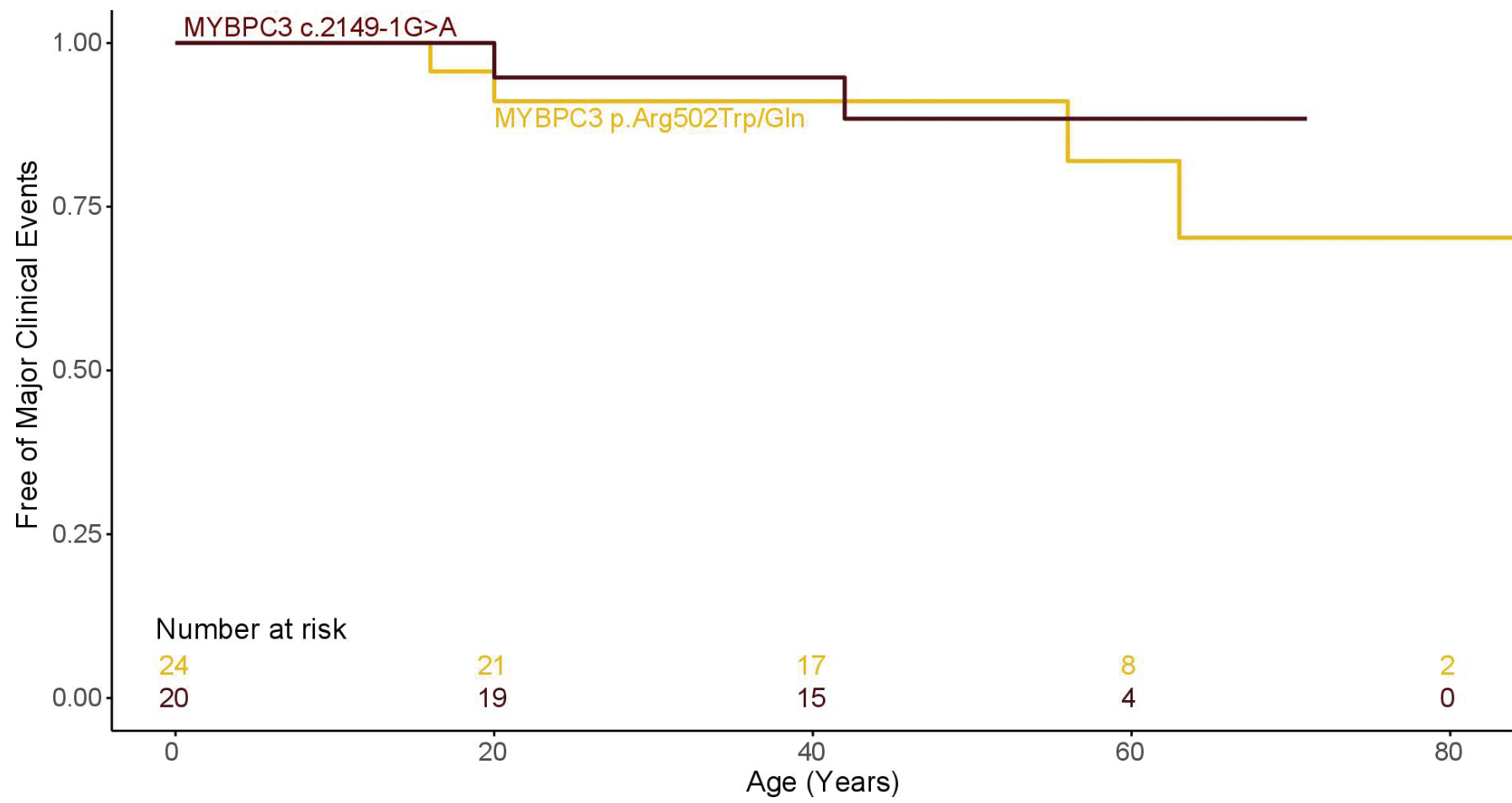

Figure 4 Survival free from major clinical outcomes. Comparison between MYBPC3 c.2149-1G>A and MYBPC3 p.Arg502Trp/ Gln variants.

major adverse outcomes was similar between the two groups. It is believed that founder mutations give rise to benign or intermediary phenotypes and disease course to withstand negative selection pressure, most of the carriers surviving the reproductive age. This allows these variants to perpetuate through multiple generations. ${ }^{30}$ Nevertheless, $20.0 \%$ of MYBPC3 c.2149-1G $>$ A affected carriers showed a severe phenotype. Also, the proportion of patients in our cohort showing a high estimated risk of SCD $(40.0 \%$ according to ESC risk score, $50.0 \%$ according to American College of Cardiology/American Heart Association guidelines) as well as the proportion of patients undergoing ICD implantation $(35.0 \%)$ and incidence of appropriate ICD shocks $(10.0 \%)$ is higher than described in other recent HCM cohorts. ${ }^{15} 1731$ Hence, the MYBPC3 c.2149-1G $>$ A variant should not be considered a benign mutation with a mild phenotype. Although variants affecting the position 502 of $M Y B P C 3$ are a frequent

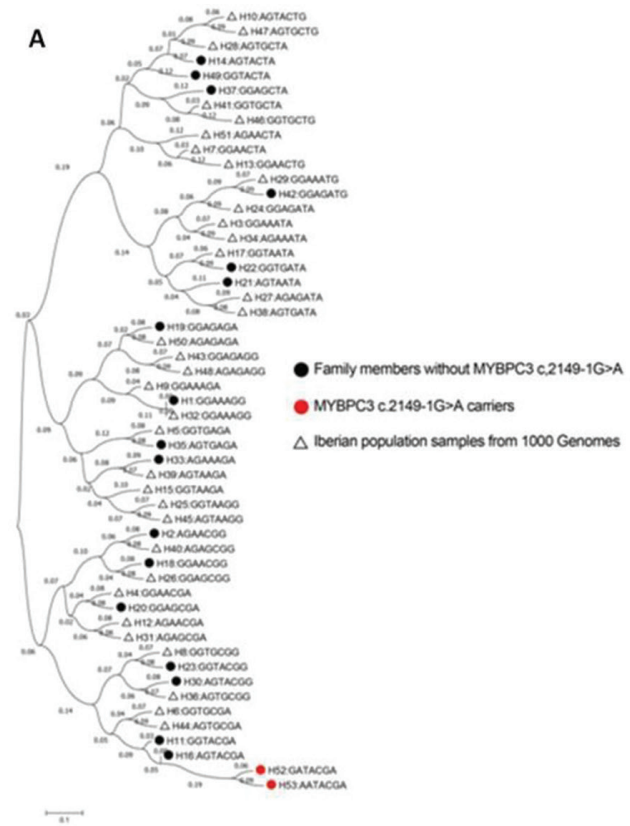

B

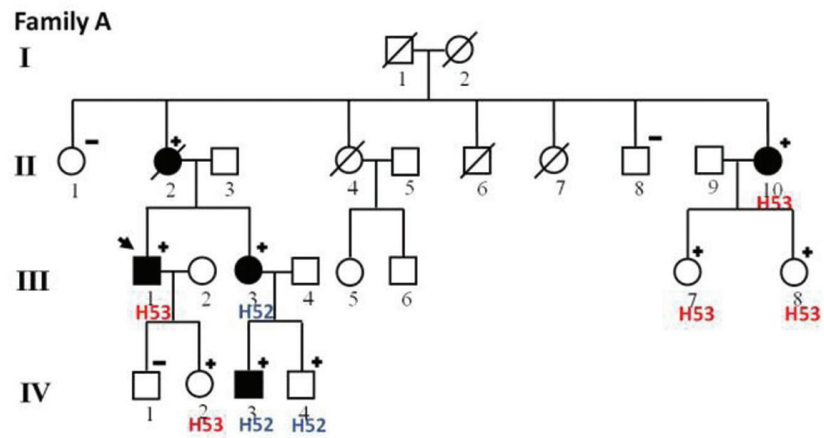

Figure 5 Haplotype reconstruction analysis. (A) phylogeny reconstruction of MYBPC3 haplotypes. Tree Neighbor-Joining in MEGA7. (B) segregation of $\mathrm{H} 52$ and $\mathrm{H} 53$ haplotypes in a family carrying MYBPC3 c.2149-1G>A. 
cause of HCM worldwide, data relating phenotype and clinical outcomes is scarce. ${ }^{89}$ Future studies with larger patient cohorts would help clarify genotype-phenotype relations for these frequent variants.

\section{LIMITATIONS}

The study is limited by the relatively small numbers of MYBPC3 c.2149-1G $>$ A affected carriers, which challenges to describe a conclusive genotype-phenotype relation for this pathogenic variant.

\section{CONCLUSIONS}

$M Y B P C 3$ c. $2149-1 \mathrm{G}>\mathrm{A}$ is a founder pathogenic variant generating an alternative splicing that leads to a truncated MYBPC3 protein. Male carriers show an early onset and a high penetrance of HCM. Carriers show better LVEF and functional class than MYBPC3 p.Arg502Trp/Gln carriers but have a similar rate of major adverse outcomes.

Contributors IMF, AIF, MÁE, SC, RL, RY, FFA and JB contributed to the design of the study. IMF, JFR, MAT and MG-M collected the data. AIF performed the statistical analysis, the molecular and haplotype analysis and the bioinformatic predictions. All authors contributed to the interpretation of the data and the review of manuscript drafts, and all approved the final manuscript. The corresponding author attests that all listed authors meet authorship criteria and that no others meeting the criteria have been omitted.

Funding The authors have not declared a specific grant for this research from any funding agency in the public, commercial or not-for-profit sectors.

Competing interests None declared.

Patient consent for publication Not required.

Ethics approval The study was approved by the Ethics Committee of the Hospital General Universitario Gregorio Marañón (Madrid, Spain) and performed in compliance with the Declaration of Helsinki.

Provenance and peer review Not commissioned; externally peer reviewed.

Data availability statement Data are available on reasonable request. All data relevant to the study are included in the article or uploaded as online supplemental information.

Open access This is an open access article distributed in accordance with the Creative Commons Attribution Non Commercial (CC BY-NC 4.0) license, which permits others to distribute, remix, adapt, build upon this work non-commercially, and license their derivative works on different terms, provided the original work is properly cited, appropriate credit is given, any changes made indicated, and the use is non-commercial. See: http://creativecommons.org/licenses/by-nc/4.0/.

\section{ORCID iDs}

Irene Méndez http://orcid.org/0000-0002-5117-4794

Marta García-Montero http://orcid.org/0000-0003-0863-8634

Javier Bermejo http://orcid.org/0000-0002-5241-2758

\section{REFERENCES}

1 Authors/Task Force members, Elliott PM, Anastasakis A, et al. 2014 ESC guidelines on diagnosis and management of hypertrophic cardiomyopathy: the task force for the diagnosis and management of hypertrophic cardiomyopathy of the European Society of cardiology (ESC). Eur Heart J 2014;35:2733-79.

2 Ommen SR, Mital S, Burke MA, et al. 2020 AHA/ACC guideline for the diagnosis and treatment of patients with hypertrophic cardiomyopathy: Executive summary: a report of the American College of Cardiology/American Heart Association joint Committee on clinical practice guidelines. Circulation 2020;142:e533-57.

3 Maron BJ. Clinical course and management of hypertrophic cardiomyopathy. N Engl J Med 2018;379:655-68.
4 Alfares AA, Kelly MA, McDermott G, et al. Results of clinical genetic testing of 2,912 probands with hypertrophic cardiomyopathy: expanded panels offer limited additional sensitivity. Genet Med 2015;17:880-8.

5 Mademont-Soler I, Mates J, Yotti R, et al. Additional value of screening for minor genes and copy number variants in hypertrophic cardiomyopathy. PLoS One 2017;12:e0181465.

6 Ho CY, Day SM, Ashley EA, et al. Genotype and lifetime burden of disease in hypertrophic cardiomyopathy: insights from the sarcomeric human cardiomyopathy registry ( $\mathrm{SHaRe})$. Circulation 2018;138:1387-98.

7 Marian AJ, Braunwald E. Hypertrophic cardiomyopathy: genetics, pathogenesis, clinical manifestations, diagnosis, and therapy. Circ Res 2017;121:749-70.

8 Page SP, Kounas S, Syrris P, et al. Cardiac myosin binding protein-C mutations in families with hypertrophic cardiomyopathy: disease expression in relation to age, gender, and long term outcome. Circ Cardiovasc Genet 2012;5:156-66.

9 Saltzman AJ, Mancini-DiNardo D, Li C, et al. Short communication: the cardiac myosin binding protein C Arg502Trp mutation: a common cause of hypertrophic cardiomyopathy. Circ Res 2010;106:1549-52.

10 Christiaans I, Nannenberg EA, Dooijes D, et al. Founder mutations in hypertrophic cardiomyopathy patients in the Netherlands. Neth Heart J 2010;18:248-54.

11 Teirlinck CH, Senni F, Malti RE, et al. A human MYBPC3 mutation appearing about 10 centuries ago results in a hypertrophic cardiomyopathy with delayed onset, moderate evolution but with a risk of sudden death. BMC Med Genet 2012;13:105.

12 van Velzen HG, Schinkel AFL, Oldenburg RA, et al. Clinical characteristics and long-term outcome of hypertrophic cardiomyopathy in individuals with a MYBPC3 (myosin-binding protein C) founder mutation. Circ Cardiovasc Genet 2017;10:1-8.

13 Jääskeläinen $P$, Miettinen R, Kärkkäinen P, et al. Genetics of hypertrophic cardiomyopathy in eastern Finland: few founder mutations with benign or intermediary phenotypes. Ann Med 2004;36:23-32.

14 Kubo T, Kitaoka H, Okawa M, et al. Lifelong left ventricular remodeling of hypertrophic cardiomyopathy caused by a founder frameshift deletion mutation in the cardiac myosin-binding protein $\mathrm{C}$ gene among Japanese. J Am Coll Cardiol 2005;46:1737-43.

15 Lorca R, Gómez J, Martín M, et al. Insights into hypertrophic cardiomyopathy evaluation through follow-up of a founder pathogenic variant. Rev Esp Cardiol 2019;72:138-44.

16 Oliva-Sandoval MJ, Ruiz-Espejo F, Monserrat L, et al. Insights into genotype-phenotype correlation in hypertrophic cardiomyopathy. findings from 18 Spanish families with a single mutation in MYBPC3. Heart 2010;96:1980-4.

17 Sabater-Molina M, Saura D, García-Molina Sáez E, et al. Nueva mutación fundadora en MYBPC3: comparación fenotípica Con La mutación de MYBPC3 más frecuente en España. Revista Española de Cardiología 2017;70:105-14.

18 Adalsteinsdottir B, Burke M, Maron BJ, et al. Hypertrophic cardiomyopathy in myosin-binding protein C (MYBPC3) Icelandic founder mutation carriers. Open Heart 2020;7:e001220-9.

19 Calore C, De Bortoli M, Romualdi C, et al. A founder MYBPC3 mutation results in $\mathrm{HCM}$ with a high risk of sudden death after the fourth decade of life. J Med Genet 2015;52:338-47.

20 Núñez L, Gimeno-Blanes JR, Rodríguez-García Ml, et al. Somatic MYH7, MYBPC3, TPM1, TNNT2 and TNNI3 mutations in sporadic hypertrophic cardiomyopathy. Circ J 2013;77:2358-65.

21 O'Mahony C, Jichi F, Pavlou M, et al. A novel clinical risk prediction model for sudden cardiac death in hypertrophic cardiomyopathy (HCM risk-SCD). Eur Heart J 2014;35:2010-20.

22 Schulz-Menger J, Bluemke DA, Bremerich J. Standardized image interpretation and post-processing in cardiovascular magnetic resonance - 2020 update: Society for Cardiovascular Magnetic Resonance (SCMR): Board of Trustees Task Force on Standardized Post-Processing. J Cardiovasc Magn Reson 2020;22:1-22.

23 Stephens M, Donnelly P. A comparison of Bayesian methods for haplotype reconstruction from population genotype data, 2003. Available: http://www.stat.washington.edu/stephens/ [Accessed 25 Mar 2020].

24 Kumar S, Stecher G, Tamura K. MEGA7: molecular evolutionary genetics analysis version 7.0 for bigger datasets. Mol Biol Evol 2016;33:1870-4.

25 Richards S, Aziz N, Bale S, et al. Standards and guidelines for the interpretation of sequence variants: a joint consensus recommendation of the American College of medical genetics and genomics and the association for molecular pathology. Genet Med 2015;17:405-23. 
26 Schlossarek S, Mearini G, Carrier L. Cardiac myosin-binding protein $\mathrm{C}$ in hypertrophic cardiomyopathy: mechanisms and therapeutic opportunities. J Mol Cell Cardiol 2011;50:613-20.

27 Zhang XL, De S, Mclntosh LP, et al. Structural characterization of the $\mathrm{C} 3$ domain of cardiac myosin binding protein $\mathrm{C}$ and its hypertrophic cardiomyopathy-related R502W mutant. Biochemistry 2014:53:5332-42.

28 Terauchi Y, Kubo T, Baba Y, et al. Gender differences in the clinical features of hypertrophic cardiomyopathy caused by cardiac myosin-binding protein $\mathrm{C}$ gene mutations. J Cardiol 2015;65:423-8.
29 Harper AR, Goel A, Grace C, et al. Common genetic variants and modifiable risk factors underpin hypertrophic cardiomyopathy susceptibility and expressivity. Nat Genet 2021;53:135-42.

30 Thompson AD, Day SM. Founder mutations in myosin-binding protein C: maybe not so benign after all. Circ Cardiovasc Genet 2017:10:e001872.

31 Maron MS, Rowin EJ, Wessler BS, et al. Enhanced American College of Cardiology/American heart association strategy for prevention of sudden cardiac death in high-risk patients with hypertrophic cardiomyopathy. JAMA Cardiol 2019;4:644-57. 